\title{
Multiple Solutions of Mixed Convective MHD Casson Fluid Flow in a Channel
}

\author{
Jawad Raza, Azizah Mohd Rohni, and Zurni Omar \\ School of Quantitative Sciences, Universiti Utara Malaysia, 06010 Sintok, Kedah, Malaysia \\ Correspondence should be addressed to Jawad Raza; jawad_6890@yahoo.com
}

Received 11 May 2016; Revised 17 July 2016; Accepted 18 July 2016

Academic Editor: Waqar A. Khan

Copyright (C) 2016 Jawad Raza et al. This is an open access article distributed under the Creative Commons Attribution License, which permits unrestricted use, distribution, and reproduction in any medium, provided the original work is properly cited.

A numerical investigation is made to determine the occurrence of the multiple solutions of MHD Casson fluid in a porous channel. Governing partial differential equation of the proposed problem converted into nonlinear ordinary differential equations by using similarity transformation. Numerical technique known as shooting method is used to investigate the existence of the multiple solutions for the variations of different parameters. Effects of physical parameters on velocity profile, temperature, concentration, and skin friction are presented in pictorial and tabulation representation.

\section{Introduction}

The fluid flow in a channel has abundant practical applications in industry from mathematical and engineering point of view [1-5]. In particular, those fluids in which the shear stresses are not linear proportional to the velocity gradient are characterized as non-Newtonian fluids which are of much interest among the researchers. Among the nonNewtonian fluids, Casson fluid has attracted more attention of researchers due to its applications in the fields of metallurgy, food processing, drilling operations, and bioengineering operations [6,7]. Some more applications of Casson fluid can be seen in the manufacturing of pharmaceutical products, coal in water, china clay, paints, synthetic lubricants, and biological fluids such as synovial fluids, sewage sludge, jelly, tomato sauce, honey, soup, and blood due to its contents such as plasma, fibrinogen, and protein [8].

Due to the novel application of MHD mixed convection flow in porous medium, in the field of industrial engineering, many researchers are attracted towards it. Design of MHD power generators, nuclear waste processing, and distribution of chemical waste control are some most conspicuous applications among all. The problem of laminar fully developed mixed convection flow in a channel was followed back to 1960 by Tao [9]. Recently, Fersadou et al. [10] investigated the problem of MHD mixed convection flow of nanofluid in a vertical porous channel numerically. Problem of mixed convection MHD flow of an $\mathrm{Al}_{2} \mathrm{O}_{3}$ water nanofluid in a channel with asymmetric heated walls was examined by Chen et al. [11]. Khan et al. [12] investigated numerically the problem of Blasius and Sakiadis flows of Casson fluid with viscous dissipation and convective boundary conditions. Umavathi and Sultana [13] talked about the mixed convective hydromagnetic flow of micropolar fluid in a vertical channel with related boundary states of third kind. Si et al. [14] utilized homotopy analysis method (HAM) to acquire the arrangement of the problem of micropolar fluid in a channel with heat and mass exchange impacts. The channel is thought to be permeable with extending/contracting dividers. Prakash and Muthtamilselvan [15] executed Crank-Nicolson limited contrast plan to take care of numerical issue of mixed convective MHD stream of micropolar fluid between two vertical permeable dividers with proper limit states of third kind. Recently, Raza et al. [16] examined the rotational effects of channel on the problem of nanofluid with shrinking channel walls.

The Casson constitutive equation was derived by Casson [17] which shows that the rate of strain and stress relationship is nonlinear. Flow of Casson fluid between two rotating cylinders is studied by Eldabe and Salwa [18]. Attia and SayedAhmed [19] considered the Couette flow of electrically conducting Casson fluid between parallel plates. Effect of mass transfer on MHD flow of Casson fluid was discussed analytically by Shehzad et al. [20]. Taylor's series was employed in 
order to solve nonlinear differential equations. Explicit finite difference method of unsteady Casson fluid flow through parallel plates was investigated by Afikuzzaman et al. [21]. Recently, Reddy et al. [22] discussed the effects of Joule heating and Hall effects on free convection in an electrically conducting Casson fluid in a vertical channel in the presence of viscous dissipation. Analytical solutions were found with the help of homotopy analysis method (HAM) and compared with Adomian Decomposition Method (ADM). Walawander et al. [23], Batra and Jena [24], Sayed Ahmed and Attia [25], Kataria and Patel [26], and Das et al. [27] have reported the flow of Casson fluid under different flow regimes. None of the investigations cited above dealt with multiple solutions of Casson fluid in a channel.

Motivated by the above-cited investigations, the aim of the present study is to investigate the multiple solutions of mixed convection flow of Casson fluid in a porous channel under the influence of the magnetic field. The effects of different parameters such as Reynold number $R$, magnetic field $M$, Casson parameter $\beta$, and bouncy parameter $\lambda$ on velocity and temperature profiles are discussed graphically and also in tabulation representation.

\section{Formulation of the Problem}

Consider the steady, incompressible MHD flow of Casson fluid in a channel. The $x$-axis is along the centerline of the channel, parallel to the channel surfaces and the $y$-axis is perpendicular to it. Lower wall of the channel is located at $y=-H$ and upper wall is at $y=H$. The fluid is injected into the channel and extracted out at a uniform velocity $V$ $(V>0$ suction and $V<0$ injection) from upper wall and lower wall, respectively. A uniform magnetic field of strength $B_{\circ}$ is applied perpendicular to the velocity field. The induced magnetic field is negligible as compared with the imposed field.

The constitutive equation for Casson fluid can be written as [17]

$$
\tau_{i j}= \begin{cases}2\left(\mu_{B}+\frac{\tau_{y}}{\sqrt{2 \pi}}\right) e_{i j}, & \pi>\pi_{c}, \\ 2\left(\mu_{B}+\frac{\tau_{y}}{\sqrt{2 \pi_{c}}}\right) e_{i j}, & \pi<\pi_{c},\end{cases}
$$

where $\mu_{B}$ is the plastic dynamic viscosity of the nonNewtonian fluid, $\tau_{y}$ is the yield stress of the fluid, $\pi$ is the product of the component of deformation rate with itself, and $\pi_{c}$ is critical value of $\pi$ based on non-Newtonian model. Under these assumptions the governing equations for MHD boundary layer flow of Casson fluid are expressed as the following equations:

$$
\begin{aligned}
\frac{\partial u}{\partial x}+\frac{\partial v}{\partial y}= & 0, \\
u \frac{\partial u}{\partial x}+v \frac{\partial u}{\partial y}= & -\frac{1}{\rho} \frac{\partial p}{\partial x}+v\left(1+\frac{1}{\beta}\right) \frac{\partial^{2} u}{\partial y^{2}}-\frac{\sigma B_{\circ}^{2} u}{\rho} \\
& \pm g\left(\beta_{T}\left(T-T_{2}\right)+\beta_{C}\left(C-C_{2}\right)\right),
\end{aligned}
$$

$$
\begin{aligned}
& u \frac{\partial v}{\partial x}+v \frac{\partial v}{\partial y}=-\frac{1}{\rho} \frac{\partial p}{\partial y}+v\left(1+\frac{1}{\beta}\right) \frac{\partial^{2} v}{\partial x^{2}} \\
& u \frac{\partial T}{\partial x}+v \frac{\partial T}{\partial y}=\frac{k}{\rho C_{p}} \frac{\partial^{2} T}{\partial y^{2}}, \\
& u \frac{\partial C}{\partial x}+v \frac{\partial C}{\partial y}=D \frac{\partial^{2} C}{\partial y^{2}}-\kappa_{1} C,
\end{aligned}
$$

where $\rho$ is density, $\mu$ is dynamic viscosity, $\nu$ is kinematic viscosity, $\sigma$ is electrical conductivity, $\beta$ is Casson fluid parameter, $T$ is temperature of the fluid, $k$ is thermal conductivity, $\kappa_{1}$ is reaction rate, $D$ is mass diffusion, and $C$ is the concentration field.

Along with boundary conditions,

$$
\begin{aligned}
u & =0, \\
v & =\frac{V}{2}, \\
T & =T_{2}, \\
C & =C_{2}, \quad \text { at } y=H, \\
\frac{\partial u}{\partial y} & =0, \\
v & =0, \\
T & =T_{1}, \\
C & =C_{1}, \quad \text { at } y=0 .
\end{aligned}
$$

Introducing stream function such that $\bar{u}=\partial \psi / \partial y, \bar{v}=$ $-\partial \psi / \partial x$ and eliminating pressure term from (3) and (4) by introducing vorticity $\omega$, we get

$$
\begin{aligned}
u \frac{\partial \omega}{\partial x}+v \frac{\partial \omega}{\partial y}= & v\left(1+\frac{1}{\beta}\right)\left(\frac{\partial^{2} \omega}{\partial x^{2}}+\frac{\partial^{2} \omega}{\partial y^{2}}\right)-\frac{\sigma B_{\circ}^{2} u^{\prime}}{\rho} \\
& \pm \frac{\partial}{\partial y}\left(g\left(\beta_{T}\left(T-T_{2}\right)+\beta_{C}\left(C-C_{2}\right)\right)\right),
\end{aligned}
$$

where $\omega=(\partial \bar{v} / \partial x-\partial \bar{u} / \partial y)$.

Define

$$
\begin{aligned}
x^{*} & =\frac{x}{H}, \\
y^{*} & =\frac{y}{H}, \\
u & =-V x^{*} f^{\prime}\left(y^{*}\right), \\
v & =V f\left(y^{*}\right), \\
\theta\left(y^{*}\right) & =\frac{T-T_{2}}{T_{1}-T_{2}}, \\
\phi\left(y^{*}\right) & =\frac{C-C_{2}}{C_{1}-C_{2}} .
\end{aligned}
$$




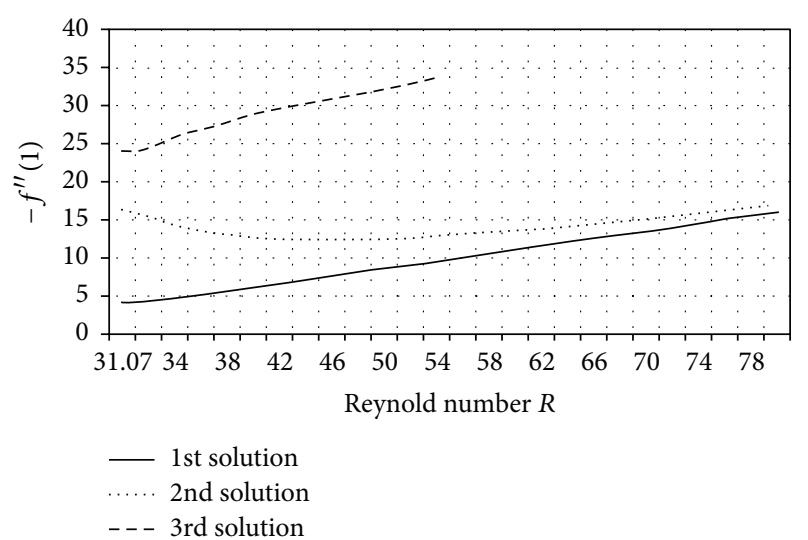

FIGURE 1: Skin friction $-f^{\prime \prime}(1)$ against the values of Reynold number $R$.

Then governing nonlinear momentum and energy equations of the proposed problem can be written as

$$
\begin{aligned}
& \left(1+\frac{1}{\beta}\right) f^{\prime \prime \prime \prime}-M^{2} f^{\prime \prime}+R\left(f^{\prime} f^{\prime \prime}-f f^{\prime \prime \prime}\right) \\
& \quad+\lambda\left(\theta^{\prime}+N \phi^{\prime}\right)=0, \\
& \theta^{\prime \prime}-\operatorname{Pr} f \theta^{\prime}=0, \\
& \varphi^{\prime \prime}-\operatorname{Sc} \gamma \varphi-\operatorname{Sc} f \varphi^{\prime}=0 .
\end{aligned}
$$

Boundary conditions (7) reduce into

$$
\begin{aligned}
f(1) & =\frac{1}{2}, \\
f^{\prime}(1) & =0, \\
\theta(1) & =0, \\
\varphi(1) & =0, \\
f^{\prime \prime}(0) & =0, \\
f(0) & =0, \\
\theta(0) & =1, \\
\varphi(0) & =1 .
\end{aligned}
$$

Here, $R=V H / v$ is Reynolds number $(R>0$ for suction $R<$ 0 and for injection), $M^{2}=\sigma B_{0}^{2} H^{2} / \mu$ is Hartman number, $\lambda=G r_{x} / R^{2}$ is the thermal buoyancy parameter, $G r_{x}=$ $V H^{4} g \beta_{T}\left(T_{1}-T_{2}\right) / x \nu^{3}$ is Grashof number, $N=\beta_{C}\left(C_{1}-\right.$ $\left.C_{2}\right) /\left(T_{1}-T_{2}\right)$ is concentration buoyancy parameter, $\operatorname{Pr}=$ $\rho C_{P} H V / \kappa$ is Prantl number, Sc $=H V / D$ is Smith number, and $\gamma=\kappa_{1} H / \nu$ is chemical reaction rate.

\section{Numerical Solution of the Problem via Shooting Method}

We employ shooting method in order to find the numerical solution of the proposed problem. For this we have to reduce the governing boundary value problem (10) into initial value problem by assuming $x_{1}=\eta, x_{2}=f, x_{3}=f^{\prime}, x_{4}=f^{\prime \prime}$, $x_{5}=f^{\prime \prime \prime}, x_{6}=\theta^{\prime}, x_{7}=\varphi^{\prime}$; then the following system is obtained

$$
\begin{aligned}
& \left(\begin{array}{c}
x_{1}^{\prime} \\
x_{2}^{\prime} \\
x_{3}^{\prime} \\
x_{4}^{\prime} \\
x_{5}^{\prime} \\
x_{6}^{\prime} \\
x_{7}^{\prime}
\end{array}\right) \\
& =\left(\begin{array}{c}
x_{3} \\
x_{4} \\
x_{5} \\
\frac{\beta}{(1+\beta)}\left(M^{2} x_{4}-R\left(x_{3} x_{4}-x_{2} x_{5}\right)-\lambda\left(x_{6}+N x_{7}\right)\right) \\
\operatorname{Pr} x_{2} x_{6} \\
\operatorname{Sc} \gamma \varphi+\operatorname{Sc} x_{2} x_{7}
\end{array}\right),
\end{aligned}
$$

with initial condition

$$
\left(\begin{array}{l}
x_{1}(1) \\
x_{2}(1) \\
x_{3}(1) \\
x_{4}(1) \\
x_{5}(1) \\
x_{6}(1) \\
x_{7}(1)
\end{array}\right)=\left(\begin{array}{c}
1 \\
\frac{1}{2} \\
0 \\
\alpha_{1} \\
\alpha_{2} \\
\alpha_{3} \\
\alpha_{4}
\end{array}\right) .
$$

Here, $\alpha_{1}, \alpha_{2}, \alpha_{3}, \alpha_{4}$ are missing initial conditions. In a shooting method, the missing (unspecified) initial condition at the initial point of the interval is assumed, and the differential equation is then integrated numerically as an initial valued problem to the terminal point. The accuracy of the assumed missing initial condition is then checked by comparing the calculated value of the dependent variable at the terminal point with its given value there. If a difference exists, another value of the missing initial condition must be assumed and the process is repeated. This process is continued until the agreement between the calculated and the given condition at the terminal point is within the specified degree of accuracy. It is important to notice that we have to shoot the values of $\alpha_{1}, \alpha_{2}, \alpha_{3}, \alpha_{4}$. Since these values are not given in the boundary condition (13), by trial and error, suitable guess values are made and integration is carried out. The details of shooting method with Maple implementation shoot have been described by Meade et al. [28].

\section{Results and Discussions}

Present section is devoted to discuss the numerical results of our finding both in tabulation and in graphical form. Our main motive is to investigate the multiple solutions of the proposed problem. We have prepared Figures 1-6 in 


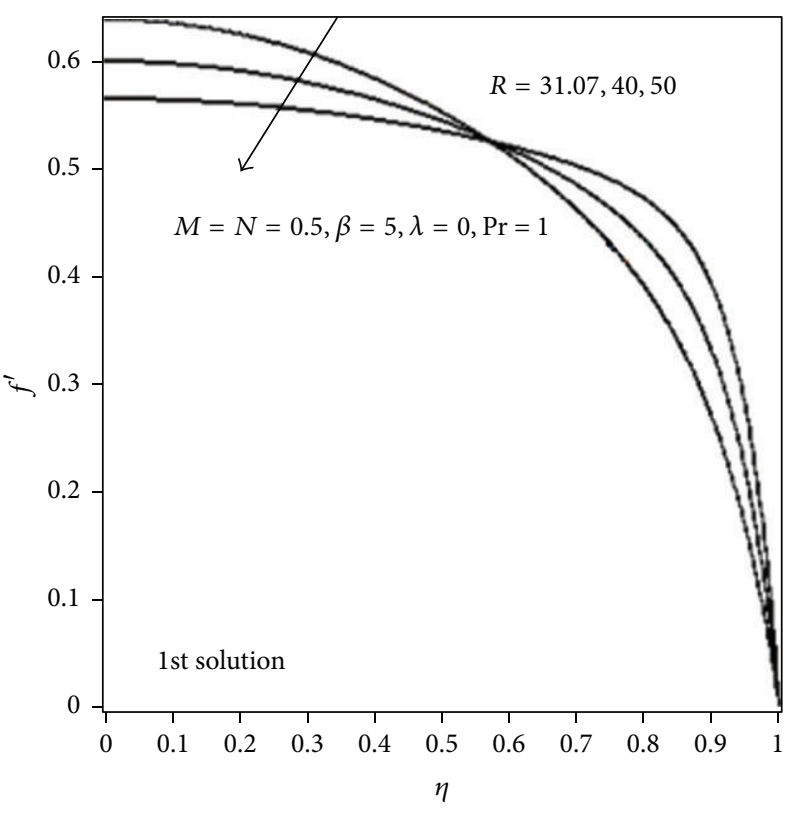

(a)

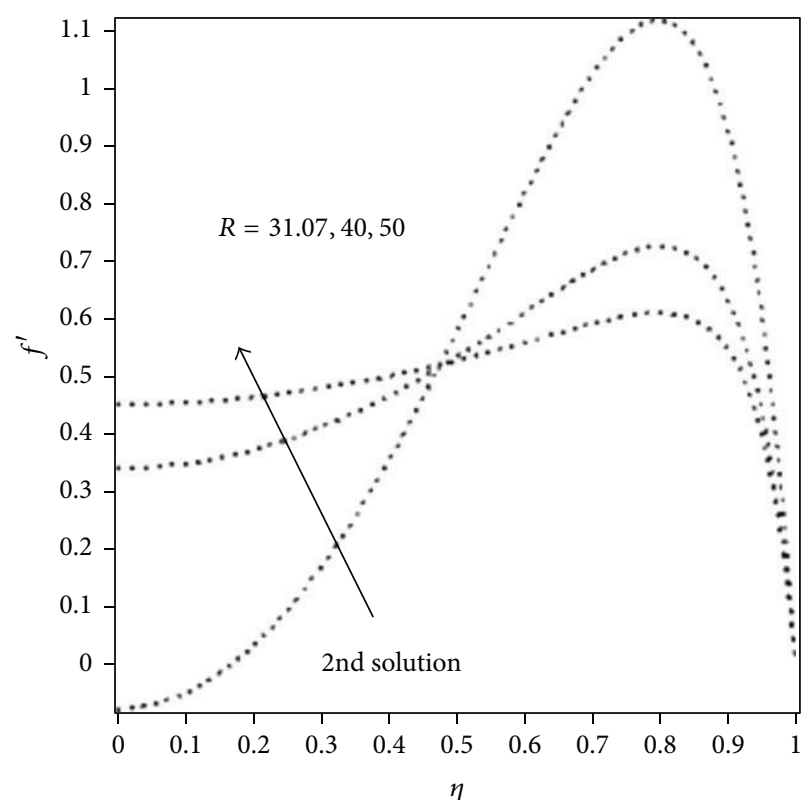

(b)

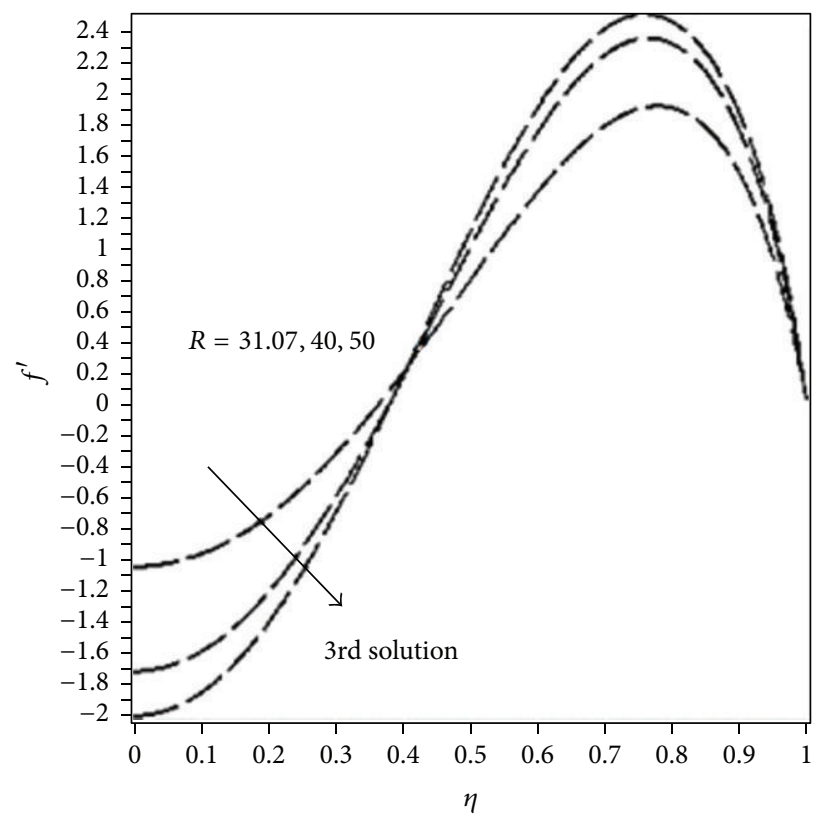

(c)

FIGURE 2: Effect of Reynold number $R$ on velocity profile $f^{\prime}(\eta)$.

order to evaluate the effects of different physical quantities on the velocity, temperature, and mass fraction. The effects include the existence of multiple solutions, Reynold number $R$, magnetic field $M$, Casson parameter $\beta$, Prandtl number $\operatorname{Pr}$, Smith number Sc, and chemical reaction rate $\gamma$.

Based on the finding of multiple solutions it is concluded that there is only one solution in the case of $R<0, \beta \in(0, \infty)$ and $\beta \in(0,5), R \in[0, \infty)$. However, there exist multiple solutions for $\beta \in[5, \infty)$ and $R \in[31.07, \infty)$ for any value of magnetic number $M \in[0,2.0]$. So we can say that there is a critical value of Casson number $\beta$ and suction parameter $R$ such that $(\beta)_{\text {critical }}=5$ and $(R)_{\text {critical }}=31.07$. So it can be defended as there is no multiple solutions if $\beta<(\beta)_{\text {critical }}$ and $<(R)_{\text {critical. }}$. The said phenomena can be observed from Figure 1. We plot the magnitude of the skin friction $\left|f^{\prime \prime}(1)\right|$ against the values of Reynold number $R$.

Effects of Reynold number $R$ on velocity profile $f^{\prime}(\eta)$ for non-buoyant flow case $\lambda=0$ are presented in Figure 2. It is noticed that with enhanced values of Reynolds number $R$, velocity profile $f^{\prime}(\eta)$ decreases near the center of the channel 


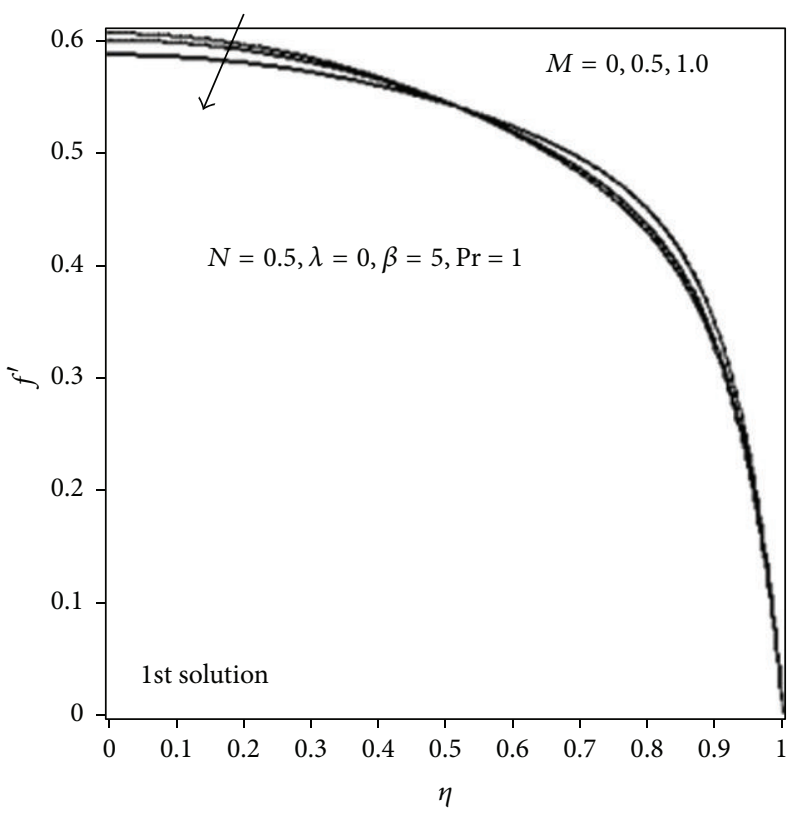

(a)

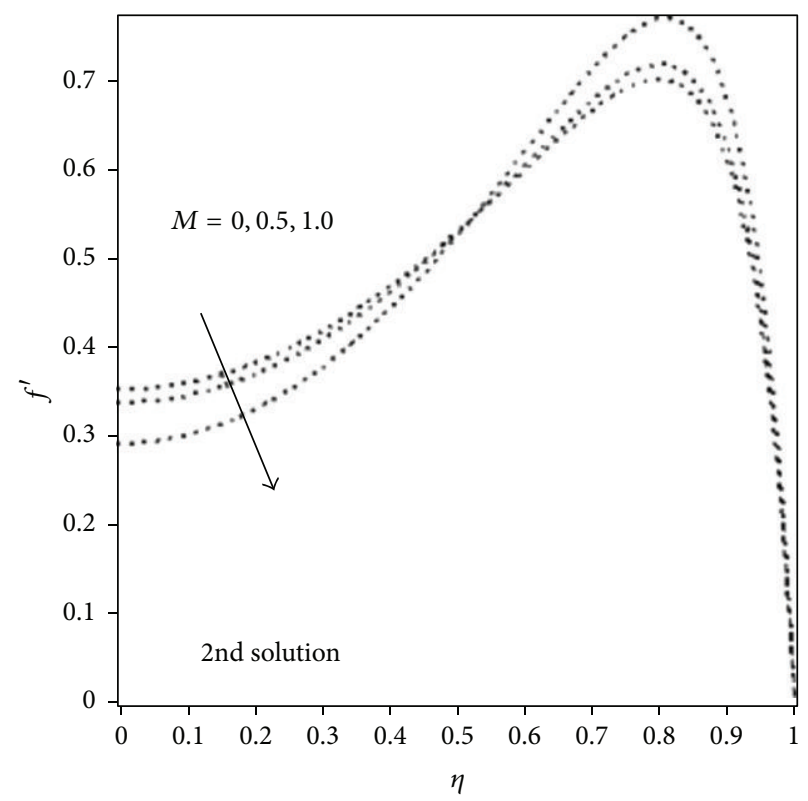

(b)

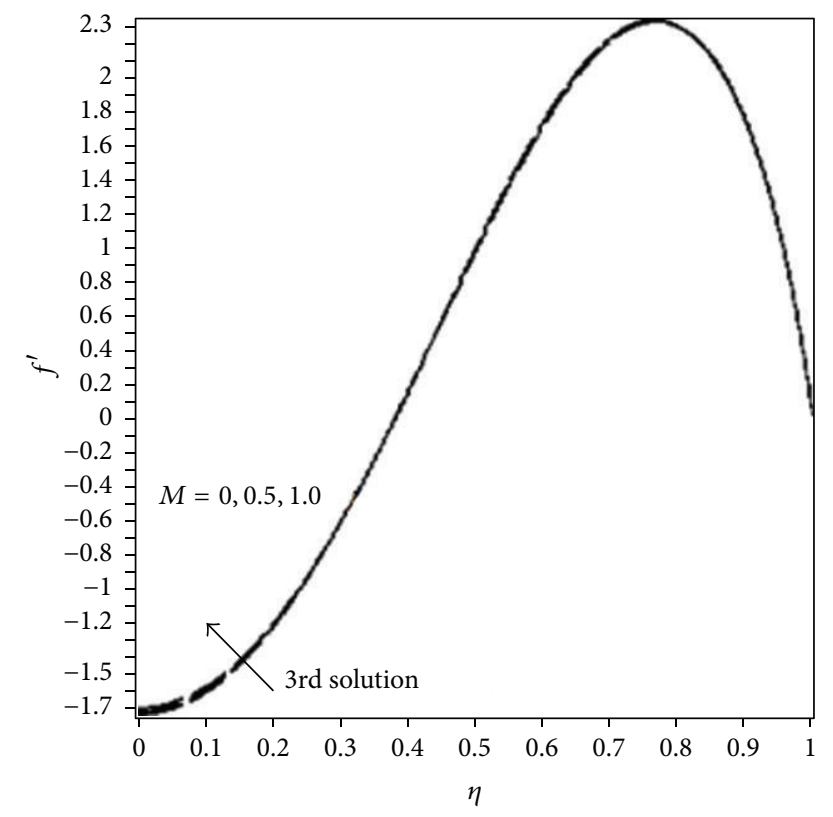

(c)

FIgURE 3: Effect of magnetic field $M$ on velocity profile $f^{\prime}(\eta)$.

for the first (1st) and third (3rd) solutions and increases near the walls of the channel. However, totally reverse behavior is observed for second (2nd) solution. Furthermore, triple solutions exist only for $R \geq 31.07$. Effect of magnetic field $M$ on velocity profile $f^{\prime}(\eta)$ corresponding to forced convection or non-buoyant flow case $\lambda=0$ is shown in Figure 3. It is concluded that velocity profile $f^{\prime}(\eta)$ for 1st and 2nd solutions decreases near the center of the channel $\eta \approx 0$ and increases near the channel walls. Since magnetic field is applied perpendicular to the channel walls, effect of magnetic field can be seen clearly near the channel walls $\eta \approx 1$. Physically we can say that magnetic field enhances the viscosity of the fluid due to the chain deformation of the fluid particles. The chain-like structure retards the flow and decelerates the motion. This results in the fact that the fluid flow can be controlled by applying magnetic field which results in many control based applications including MHD power generation, casting of metals, blood flow in arteries, and many, many more. Effect of Casson parameter $\beta$ on velocity profile $f^{\prime}(\eta)$ for the case of forced convection $\lambda=$ 0 is depicted in Figure 4. Velocity profile $f^{\prime}(\eta)$ decreases gradually for the 1st and 3rd solutions near the center of 


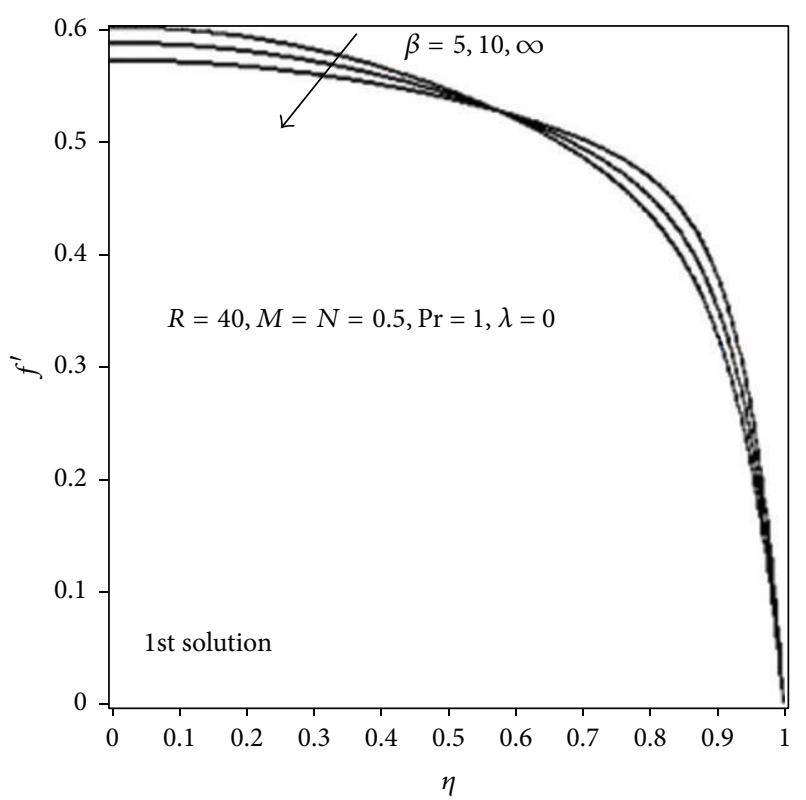

(a)

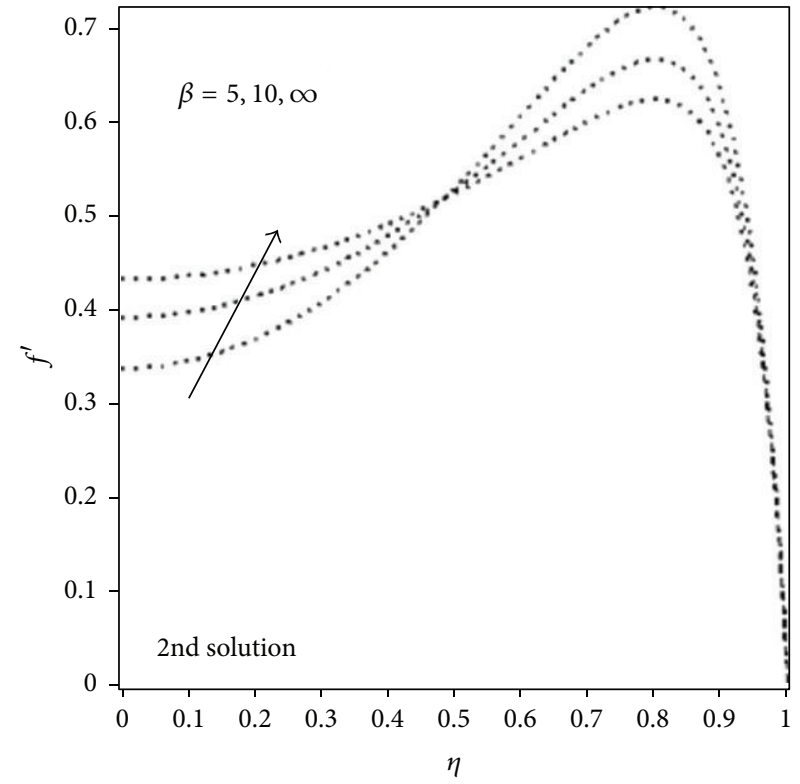

(b)

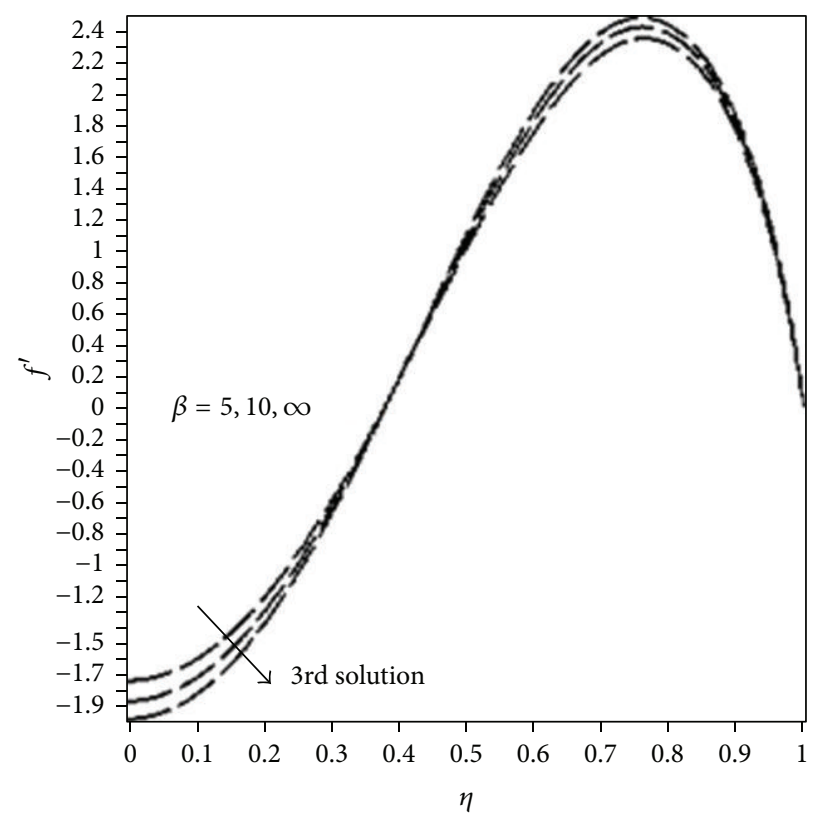

(c)

FIGURE 4: Effect of Casson parameter $\beta$ on velocity profile $f^{\prime}(\eta)$.

the channel and increases near the channel wall $\eta \approx 1$. However, velocity increases for the 2nd solution as the enhancement of the Casson parameter $\beta$ is taking place in the neighborhood of $\eta \approx 0$. Furthermore, $\beta \rightarrow \infty$ corresponds to the Newtonian fluid. This is because of the fact that fluid is extracted from the walls of the channel with constant velocity that decreases the viscosity of the fluid particles which results in increase in the velocity near the wall of the channel. Figure 5 presented the effect of Prandtl number Pr on temperature profile $\theta(1)$ for non-buoyant $\lambda=0$ case.
It is concluded from these profiles that temperature profile $\theta(1)$ increases strictly monotonically as the Prandtl number Pr increases for 1st and 2nd solutions. On the other hand, temperature profile $\theta(1)$ decreases as the Prandtl number $\mathrm{Pr}$ increases for 3rd solution. Effects of Smith number Sc on concentration profile $\phi(\eta)$ for $\lambda=0$ (non-buoyant case) are plotted in Figure 6. Concentration profile $\phi(\eta)$ decreases by the increase of the strength of Smith number Sc for all triple solutions. Physically it can be argued that an increase in Smith number Sc prompts the increment in the quantity of 


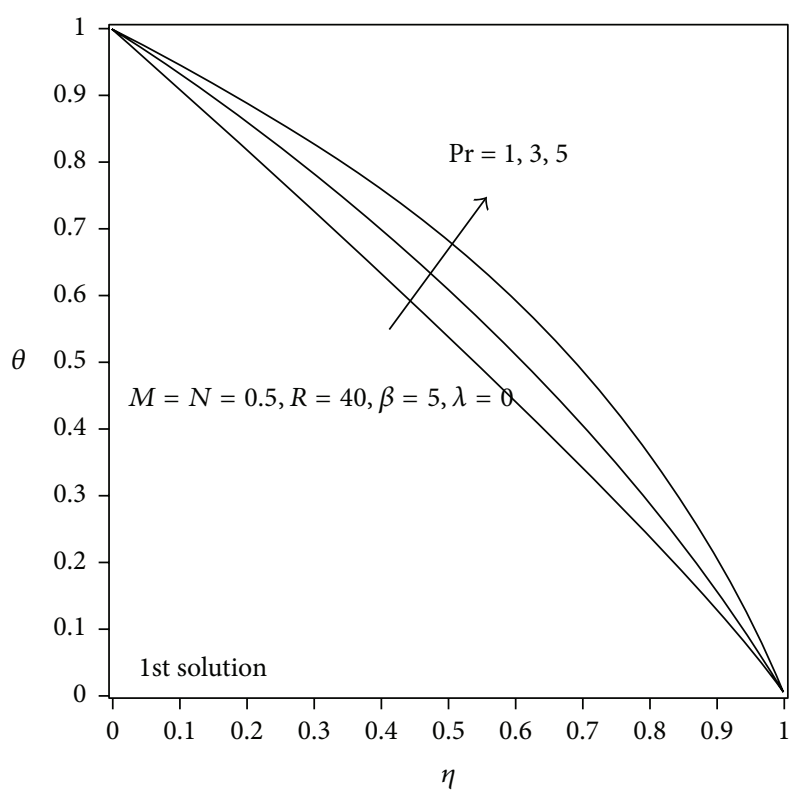

(a)

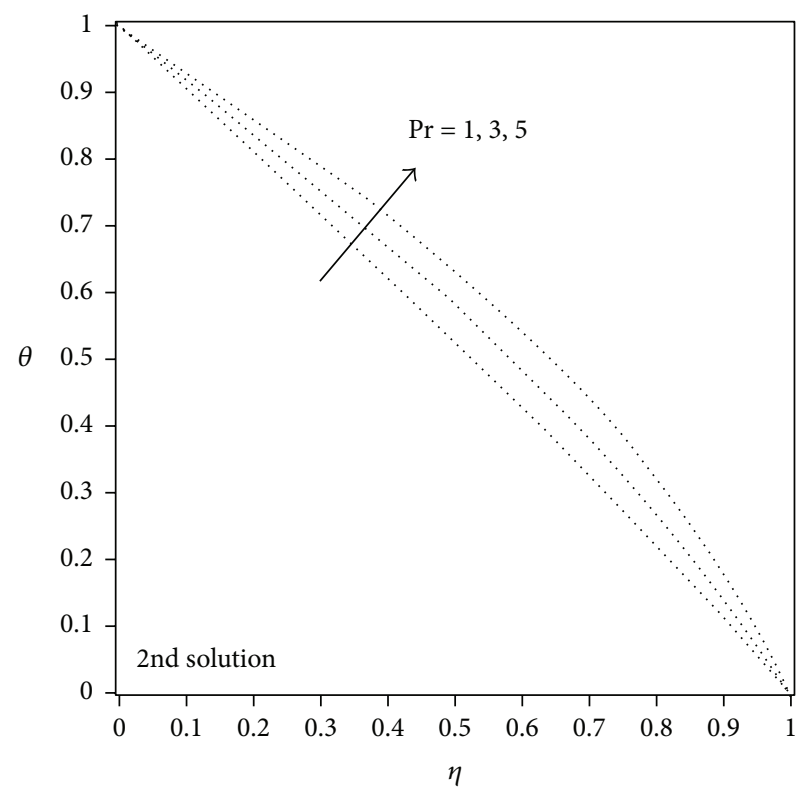

(b)

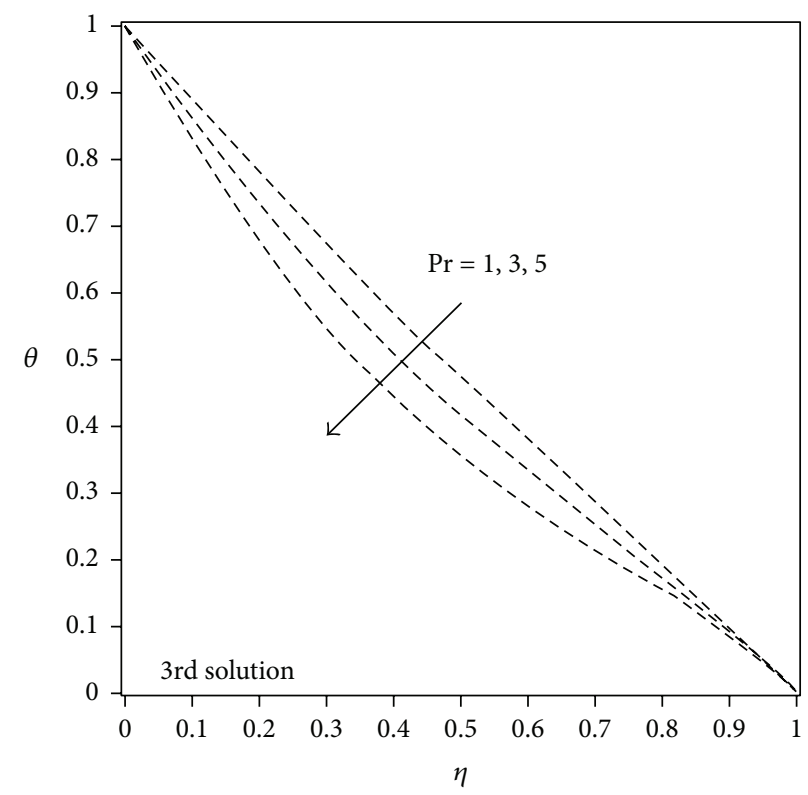

(c)

Figure 5: Effect of Prandtl number Pr on temperature profile $\theta(\eta)$.

solute atoms experiencing substance reaction coming about the way that concentration of the fluid's molecule diminishes monotonically. In Figure 7, we plot $\theta^{\prime}(1)$ against the values of Reynold number $R$ for the case of forced convection $\lambda=0$. It is seen that 1st and 2nd solutions overlap each other as the Reynold number $R$ increases.

In Tables 1 and 2, we represented numerical values of skin friction $f^{\prime \prime}(1)$ and $\theta^{\prime}(1)$ for the variations of different physical parameters. Table 1 presented the numerical values of skin friction $f^{\prime \prime}(1)$ for the variation of buoyancy parameter $\lambda$ by fixing $R=36, M=N=0.5, \beta=5, \operatorname{Pr}=1$, $\mathrm{Sc}=1, \gamma=1.2$. Magnitude of the skin friction increases for
TABLE 1: Skin friction for different values of buoyancy parameters for $R=36, M=N=0.5, \beta=5, \operatorname{Pr}=1, \mathrm{Sc}=1, \gamma=1.2$.

\begin{tabular}{lccc}
\hline$\lambda$ & 1st solution & 2nd solution & 3rd solution \\
& $f^{\prime \prime}(1)$ & $f^{\prime \prime}(1)$ & $f^{\prime \prime}(1)$ \\
\hline-0.50 & -3.29405873 & -14.78794525 & -27.20927012 \\
-0.25 & -4.19831393 & -14.22940191 & -27.20983823 \\
0 & -5.21362887 & -13.55733409 & -27.21039669 \\
0.25 & -6.42985179 & -12.68188468 & -27.21094599 \\
0.50 & -8.22263120 & -11.22740570 & -27.21148660 \\
\hline
\end{tabular}




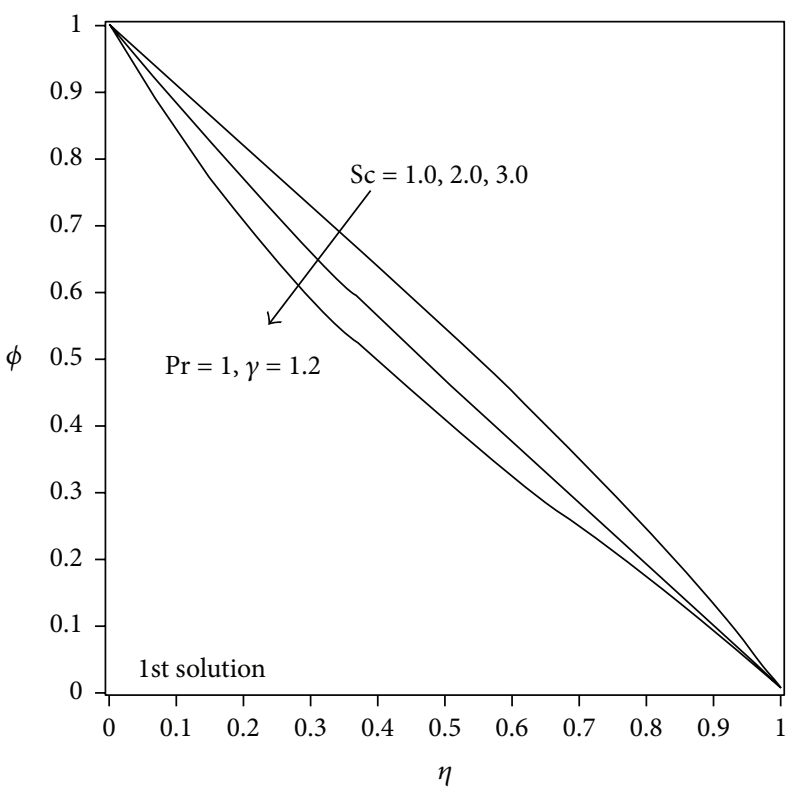

(a)

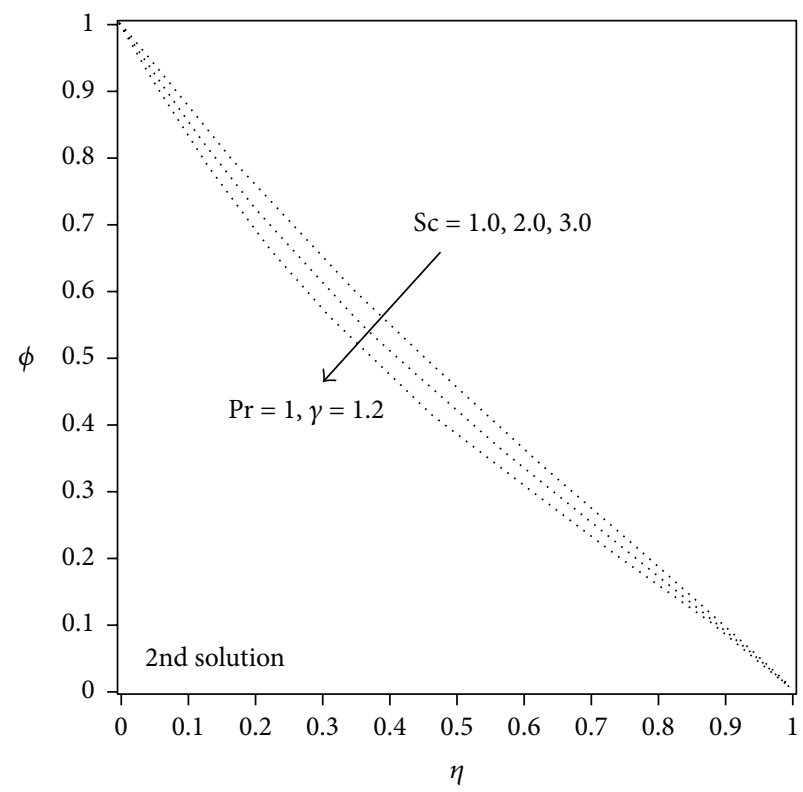

(b)

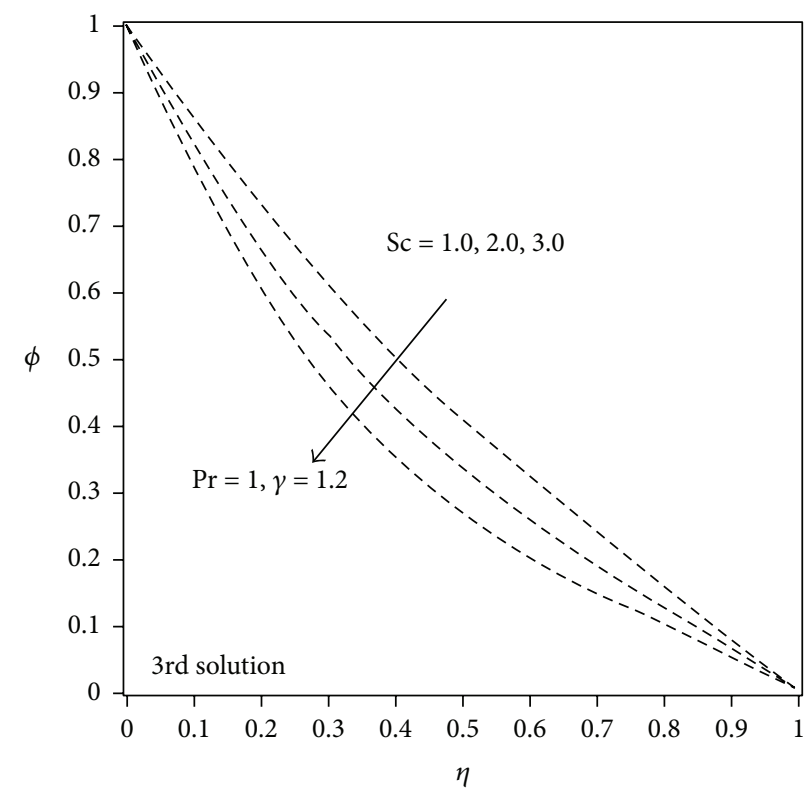

(c)

Figure 6: Effect of Smith number Sc on concentration profile $\phi(\eta)$ for $\lambda=0$.

the 1st and 3rd solutions and decreases for 2 nd solution in the case of opposing flow $\lambda<0$. Furthermore, same behavior is depicted for the case of assisting flow $\lambda>0$; therefore the fluid velocity near the channel walls $\eta \approx 1$ increases. From Table 2 it is concluded that magnitude of skin friction $f^{\prime \prime}(1)$ and $\theta^{\prime}(1)$ increases and decreases, respectively, as Reynold number increases for the case of assisting flow $\lambda>0$ only for 1st and 3rd solutions by setting $M=N=0.5, \operatorname{Pr}=1, \mathrm{Sc}=2$, $\gamma=1.2$. Numerical values of skin friction $f^{\prime \prime}(1)$ increase for 1st and 3rd solutions and decrease for 2 nd solution by the variation of Reynolds number for the case of opposing flow $\lambda<0$ by setting $M=N=0.5, \operatorname{Pr}=1, \mathrm{Sc}=2, \gamma=1.2$.

\section{Conclusion}

Multiple solutions of MHD Casson fluid flow in a channel with heat and mass transfer are analyzed. Here are some important observations have been engendered in the light of numerical investigation:

(i) There is only single solution for the case of injection $R<0$ for any value of Casson number $\beta$ or Hartman number $M$.

(ii) There exist multiple solutions only for $\beta \in[5, \infty)$ and $R \in[31.07, \infty)$ for any value of magnetic number $M \in[0,2.0]$. 
TABLE 2: Skin friction and temperature gradient for different values of Reynold number $R$.

\begin{tabular}{lccccccc}
\hline \multirow{2}{*}{$\lambda$} & \multirow{2}{*}{ 1st solution } & 2nd solution & 3rd solution & 1st solution & \multicolumn{2}{c}{ 2nd solution } & 3rd solution \\
& & $f^{\prime \prime}(1)$ & $f^{\prime \prime}(1)$ & $f^{\prime \prime}(1)$ & $\theta^{\prime}(1)$ & $\theta^{\prime}(1)$ & -1.12415316 \\
\hline 31.07 & & -3.56529871 & -16.7538394 & -23.89501535 & -1.20630237 & -1.04645775 \\
35 & -0.25 & -4.06923819 & -14.50163765 & -26.71544153 & -1.20492033 & -1.14909835 & -1.01703703 \\
40 & & -4.70569981 & -13.72648291 & -28.87613992 & -1.20354537 & -1.16169799 & -0.99886321 \\
\hline 31.07 & & -4.22815985 & -16.33685245 & -23.92995344 & -1.20259758 & -1.12723885 & -1.045737 \\
35 & 0 & -5.00282319 & -13.89608196 & -26.71780315 & -1.20017552 & -1.15257708 & -1.01679981 \\
40 & & -6.10401248 & -12.73103850 & -28.87353590 & -1.19720323 & -1.16644810 & -0.99874016 \\
\hline 31.07 & & -4.93400210 & -15.87676698 & -23.96319996 & -1.19862360 & -1.13057525 & -1.04504186 \\
35 & 0.25 & -6.08426152 & -13.14036724 & -26.72012735 & -1.19463415 & -1.15685896 & -1.01656453 \\
40 & & -8.28517017 & -10.94971752 & -28.87095120 & -1.18721976 & -1.17484905 & -0.99861750 \\
\hline
\end{tabular}

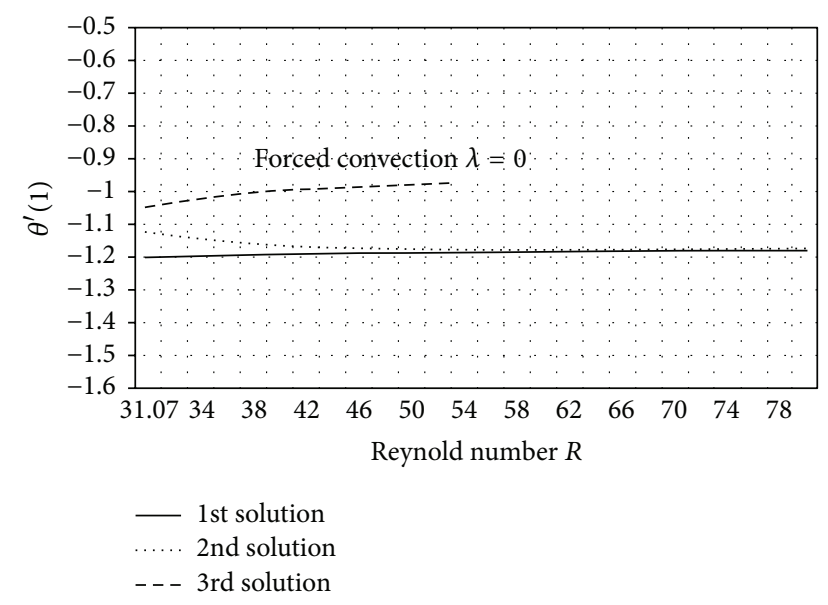

Figure 7: Effect of Reynold number $R$ on $\theta^{\prime}(1)$.

(iii) Effect of Casson number $\beta \geq 5$ and Reynolds number $R>31.06$ on velocity profile $f^{\prime}(\eta)$ increases for 1st and 3 rd solutions near the channel wall $\eta \approx 1$.

(iv) Effect of Reynold number $R$ on skin friction $f^{\prime \prime}(1)$ and $\theta^{\prime}(1)$ is the same for assisting flow $\lambda>0$ and opposing flow $\lambda<0$.

\section{Competing Interests}

The authors declare that they have no competing interests.

\section{References}

[1] B. Li, L. Zheng, X. Zhang, and L. Ma, "The multiple solutions of laminar flow in a uniformly porous channel with suction/injection," Advanced Studies in Theoretical Physics, vol. 2, no. 10, pp. 473-478, 2008.

[2] X. Si, L. Zheng, X. Zhang, and Y. Chao, "The flow of a micropolar fluid through a porous channel with expanding or contracting walls," Central European Journal of Physics, vol. 9, no. 3, pp. 825-834, 2011.

[3] X.-Y. Si, X.-H. Si, L.-C. Zheng, and X.-X. Zhang, "Homotopy analysis solution for micropolar fluid flow through porous channel with expanding or contracting walls of different permeabilities," Applied Mathematics and Mechanics-English Edition, vol. 32, no. 7, pp. 859-874, 2011.

[4] B. J. Gireesha, G. S. Roopa, and C. S. Bagewadi, "Unsteady flow and heat transfer of a dusty fluid through a rectangular channel," Mathematical Problems in Engineering, vol. 2010, Article ID 898720, 17 pages, 2010.

[5] Mahesha, B. J. Gireesha, G. K. Ramesha, and C. S. Bagewadi, "Unsteady flow of a dusty fluid through a channel having triangular cross-section in Frenet frame field system," Acta Universitatis Apulensis, vol. 25, pp. 53-75, 2011.

[6] K. Ramesh and M. Devakar, "Some analytical solutions for flows of Casson fluid with slip boundary conditions," Ain Shams Engineering Journal, vol. 6, no. 3, pp. 967-975, 2015.

[7] D. McDonald, Blood Flows in Arteries, Arnold, London, UK, 2nd edition, 1974.

[8] E. W. Merrill, A. M. Benis, E. R. Gilliland, T. K. Sherwood, and E. W. Salzman, "Pressure-flow relations of human blood in hollow fibers at low flow rates," Journal of Applied Physiology, vol. 20, no. 5, pp. 954-967, 1965.

[9] L. N. Tao, "On combined free and forced convection in Channels," Journal of Heat Transfer, vol. 82, no. 3, pp. 233-238, 1960.

[10] I. Fersadou, H. Kahalerras, and M. El Ganaoui, "MHD mixed convection and entropy generation of a nanofluid in a vertical porous channel," Computers \& Fluids, vol. 121, pp. 164-179, 2015.

[11] C.-K. Chen, B.-S. Chen, and C.-C. Liu, "Entropy generation in mixed convection magnetohydrodynamic nanofluid flow in vertical channel," International Journal of Heat and Mass Transfer, vol. 91, pp. 1026-1033, 2015.

[12] S. I. Khan, U. Khan, N. Ahmed, S. U. Jan, A. Waheed, and S. T. Mohyud-Din, "Effects of viscous dissipation and convective boundary conditions on Blasius and Sakiadis problems for Casson fluid," National Academy Science Letters, vol. 38, no. 3, pp. 247-250, 2015.

[13] J. C. Umavathi and J. Sultana, "Mixed convective flow of a micropolar fluid mixture in a vertical channel with boundary conditions of the third kind," Journal of Engineering Physics and Thermophysics, vol. 85, no. 4, pp. 895-908, 2012.

[14] X. Si, L. Zheng, P. Lin, X. Zhang, and Y. Zhang, "Flow and heat transfer of a micropolar fluid in a porous channel with expanding or contracting walls," International Journal of Heat and Mass Transfer, vol. 67, pp. 885-895, 2013. 
[15] D. Prakash and M. Muthtamilselvan, "Effect of radiation on transient MHD flow of micropolar fluid between porous vertical channel with boundary conditions of the third kind," Ain Shams Engineering Journal, vol. 5, no. 4, pp. 1277-1286, 2014.

[16] J. Raza, A. M. Rohni, Z. Omar, and M. Awais, "Heat and mass transfer analysis of MHD nanofluid flow in a rotating channel with slip effects," Journal of Molecular Liquids, vol. 219, pp. 703708, 2016.

[17] M. Casson, "A flow equation for pigment-oil suspensions of the printing ink type," in Rheology of Disperse Systems, C. C. Mills, Ed., pp. 84-104, Pergamon, Oxford, NY, USA, 1959.

[18] N. T. M. Eldabe and M. G. E. Salwa, "Heat transfer of MHD nonNewtonian Casson fluid flow between two rotating cylinders," Journal of the Physical Society of Japan, vol. 64, pp. 41-64, 1995.

[19] H. A. Attia and M. E. Sayed-Ahmed, "Transient MHD couette flow of a Casson fluid between parallel plates with heat transfer," Italian Journal of Pure and Applied Mathematics, vol. 27, pp. 1938, 2010.

[20] S. A. Shehzad, T. Hayat, M. Qasim, and S. Asghar, "Effects of mass transfer on MHD flow of Casson fluid with chemical reaction and suction," Brazilian Journal of Chemical Engineering, vol. 30, no. 1, pp. 187-195, 2013.

[21] M. Afikuzzaman, M. Ferdows, and M. M. Alam, "Unsteady MHD casson fluid flow through a parallel plate with hall current," Procedia Engineering, vol. 105, pp. 287-293, 2015.

[22] C. Reddy, C. V. Rao, and O. Surender, "Soret, joule heating and hall effects on free convection in a casson fluid saturated porous medium in a vertical channel in the presence of viscous dissipation," Procedia Engineering, vol. 127, pp. 1219-1226, 2015.

[23] W. P. Walawender, T. Y. Chen, and D. F. Cala, "An approximate casson fluid model for tube flow of blood," Biorheology, vol. 12, no. 2, pp. 111-119, 1975.

[24] R. L. Batra and B. Jena, "Flow of a Casson fluid in a slightly curved tube," International Journal of Engineering Science, vol. 29, no. 10, pp. 1245-1258, 1991.

[25] M. E. Sayed Ahmed and H. A. Attia, "Magnetohydrodynamic flow and heat transfer of a non-Newtonian fluid in an eccentric annulus," Canadian Journal of Physics, vol. 76, no. 5, pp. 391-401, 1998.

[26] H. R. Kataria and H. R. Patel, "Radiation and chemical reaction effects on MHD Casson fluid flow past an oscillating vertical plate embedded in porous medium," Alexandria Engineering Journal, vol. 55, pp. 583-595, 2016.

[27] M. Das, R. Mahato, and R. Nandkeolyar, "Newtonian heating effect on unsteady hydromagnetic Casson fluid flow past a flat plate with heat and mass transfer," Alexandria Engineering Journal, vol. 54, no. 4, pp. 871-879, 2015.

[28] D. B. Meade, B. S. Haran, and R. E. White, "The shooting technique for the solution of two-point boundary value problems," Maple Technical Newsletter, vol. 3, pp. 1-8, 1996. 


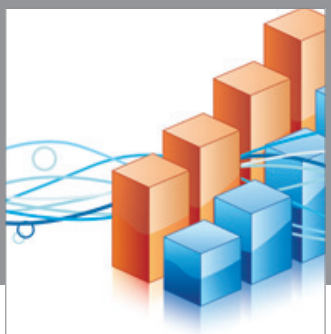

Advances in

Operations Research

vatem alat4

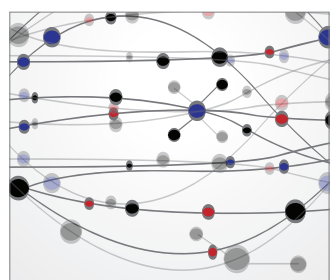

\section{The Scientific} World Journal
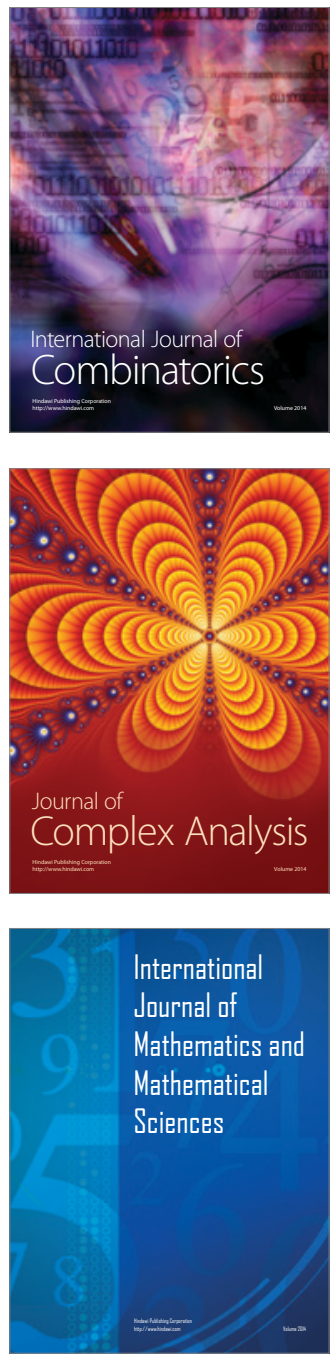
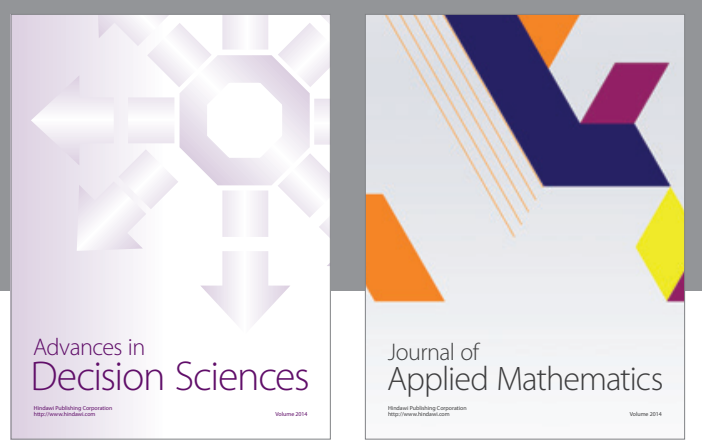

Algebra

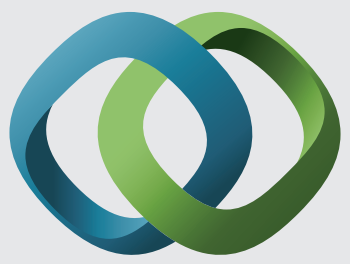

\section{Hindawi}

Submit your manuscripts at

http://www.hindawi.com
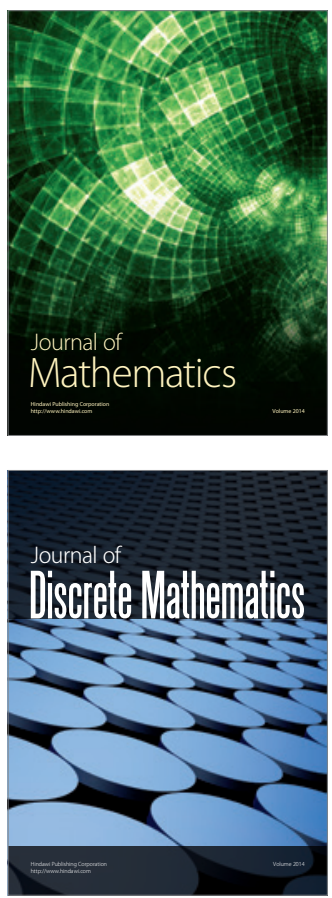

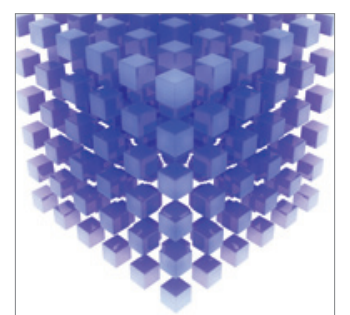

Mathematical Problems in Engineering
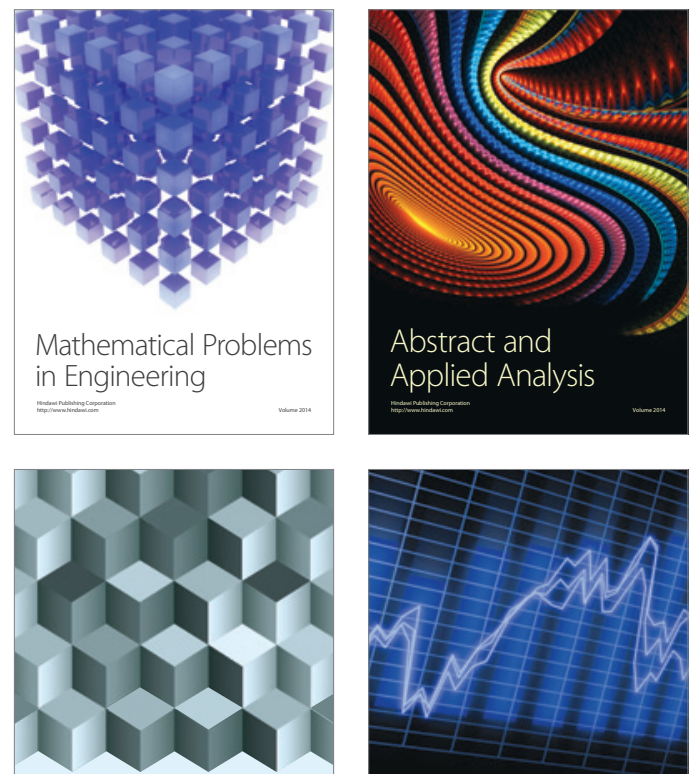

Journal of

Function Spaces

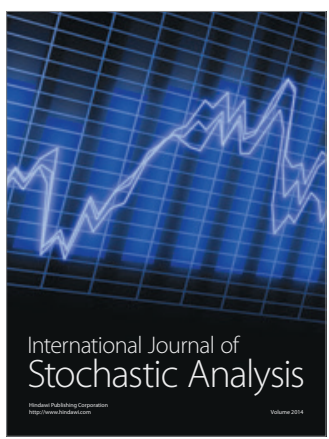

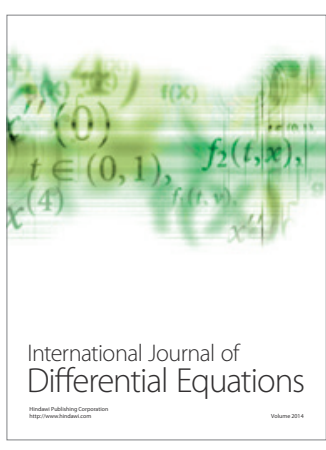
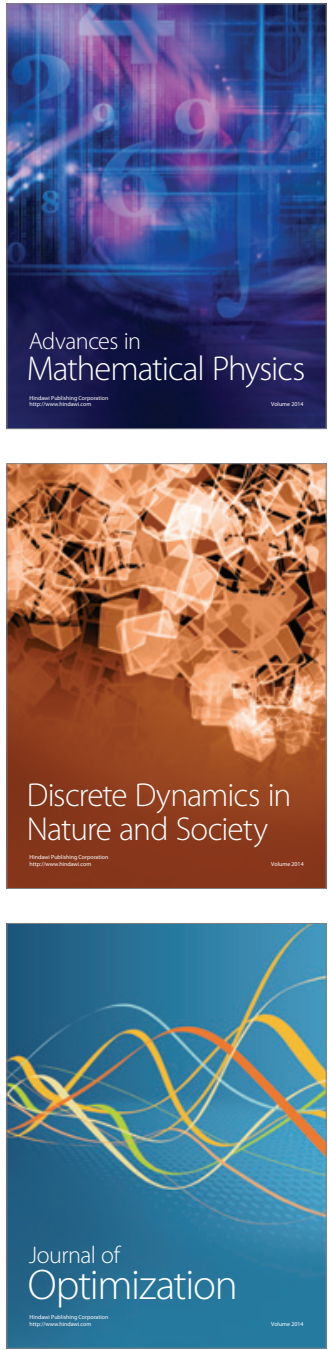